\title{
1 AtPQT11, a P450 enzyme, detoxifies paraquat via N-demethylation
}

2 Yi-Jie Huang ${ }^{1}$, Yue-Ping Huang ${ }^{1,2}$, Jin-Qiu Xia ${ }^{1}$, Zhou-Ping Fu ${ }^{3}$, Yi-Fan Chen ${ }^{3}$,

3 Yi-Peng Huang ${ }^{3}$, Aimin $\mathrm{Ma}^{4}$,Wen-Tao Hou ${ }^{1}$, Yu-Xing Chen ${ }^{1}$, Xiaoquan Qi ${ }^{4}$, Li-Ping

$4 \quad \mathrm{Gao}^{3}$, Cheng-Bin Xiang ${ }^{1}$

5

$6 \quad{ }^{1}$ School of Life Sciences, Division of Molecular \& Cell Biophysics, Hefei National

7 Science Center for Physical Sciences at the Microscale, University of Science and

8 Technology of China, The Innovation Academy of Seed Design, Chinese Academy of

9 Sciences, Hefei, Anhui Province 230027, China

$10{ }^{2}$ School of Life Sciences, Sun Yat Sen University, No. 135, Xingang Xi Road,

11 Guangzhou, 510275, P. R. China

$12{ }^{3}$ School of Life Science, Anhui Agricultural University, 130 West Changjiang Rd,

13 Hefei 230036, Anhui, China

$14{ }^{4}$ Key Laboratory of Plant Molecular Physiology, Institute of Botany, The Innovation

15 Academy of Seed Design, Chinese Academy of Sciences, Beijing, China

16

17 Correspondence:

18 Cheng-Bin Xiang

19 Email: xiangcb@ustc.edu.cn

20 orcid.org/0000-0002-7152-1458

21
Li-Ping Gao

E-mail: gaolp62@126.com 


\section{Abstract}

Paraquat is one of the most widely used nonselective herbicides in agriculture. Due to its wide use, paraquat resistant weeds have emerged and is becoming a potential threat to agriculture. The molecular mechanisms of paraquat resistance in weeds remain largely unknown. Physiological studies indicated that the impaired translocation of paraquat and enhanced antioxidation could improve paraquat resistance in plants. However, the detoxification of paraquat via active metabolism by plants has not been reported to date. Here we report that an activated expression of At1g01600 encoding the $\mathrm{P} 450$ protein CYP86A4 confers paraquat resistance as revealed by the gain-of-function mutant paraquat tolerance $11 D$ (pqt11D), in which a T-DNA with four 35S enhancers was inserted at $1646 \mathrm{bp}$ upstream the ATG of At1g01600. The paraquat resistance can be recapitulated in Arabidopsis wild type by overexpressing AtPQT11 (At1g01600), while its knockout mutant is hypersensitive to paraquat. Moreover, AtPQT11 also confers paraquat resistance in E. coli when overexpressed. We further demonstrate that AtPQT11 has P450 enzyme activity that converts paraquat to $\mathrm{N}$-demethyl paraquat nontoxic to Arabidopsis, therefore detoxifying paraquat in plants. Taken together, our results unequivocally demonstrate that AtPQT11/ CYP86A4 detoxifies paraquat via active metabolism, thus revealing a novel molecular mechanism of paraquat resistance in plants and providing a means potentially enabling crops to resist paraquat.

Key words: paraquat, paraquat resistance, AtPQT11, pqt11, CYP86A4, P450, herbicide, demethylation. 


\section{Introduction}

Paraquat (1,1'-dimethyl-4,4'-bipyridynium dichloride) has been widely used as a broad spectrum nonselective herbicide in agriculture for decades ${ }^{1}$. Plants absorb paraquat from their environment and transport to chloroplasts, where it competes for electrons on the PSI and generates superoxide that is converted to $\mathrm{H}_{2} \mathrm{O}_{2}$ by superoxide dismutases (SODs), resultantly accumulating a large amount of ROS, which may lead to cell death ${ }^{2}$.

Paraquat resistant weeds have emerged after the wide use of paraquat in agriculture. The underlying mechanisms of paraquat resistance in weeds have been studied mostly at physiological and biochemical levels ${ }^{3-6}$. From these studies, two major mechanisms of paraquat resistance were suggested, including defects in paraquat translocation restricting the transport of paraquat to chloroplasts ${ }^{4,7}$, and enhanced antioxidation scavenging ROS more efficiently ${ }^{8,9}$. However, no genetic or molecular evidence for the proposed mechanisms has been revealed in weeds. With the power of the model plant Arabidopsis, several research groups have isolated paraquat resistant mutants, originally aiming at identifying novel components in oxidative stress response or cell death ${ }^{10,11}$. These studies with Arabidopsis paraquat resistant mutants helped reveal the molecular mechanisms of paraquat resistance proposed by weed scientists based on their studies on paraquat resistant weeds. The unraveled molecular mechanisms from these studies fall into two categories; one involves paraquat uptake and transport ${ }^{12-15}$, and the other is the enhanced ability scavenging $\operatorname{ROS}^{10,11,16,17}$.

However, no evidence of paraquat degradation by plant active metabolism has been reported. Herbicide degradation by plants via active metabolism is a desirable mechanism for engineering crop resistance to herbicides. CYP450s (cytochrome P450s) constitute the biggest plant protein family and can mediate redox reaction in plant. They usually function as demethylase and/or hydroxylase in herbicide metabolism ${ }^{18}$. Some P450s metabolize herbicides to lower toxic products which are transferred to vacuole as glucosyl conjugates ${ }^{19}$. Two plant cytochrome P450 enzymes, 
CYP71A10 from Helianthus tuberosus and CYP76B1 from soybean, were reported having urea herbicide metabolism activity ${ }^{20}$. In addition, tobacco CYP81B2 and CYP71A11 catabolize chlortoluron by ring methyl hydroxylation and $\mathrm{N}-$-methylation 21.

We previously isolated a number of paraquat tolerant Arabidopsis mutants and reported pqt24 and pqt3 ${ }^{15,16}$. Here we continue to characterize those paraquat tolerance mutants by focusing on pqt11D in this study. We isolated pqt11D as a gain-of-function mutant with enhanced paraquat tolerance and identified that the At1G01600 (AtPQT11) was activated by the enhancers of the T-DNA insertion, which caused paraquat resistance in the mutant. The mutant phenotype could be recapitulated by overexpressing AtPQT11, encoding the protein CYP86A4, a member of CYP450 super family, in the wild type Arabidopsis. Further analyses revealed that AtPQT11/CYP86A4 could convert paraquat to N-demethyl paraquat in vitro by the mean of N-demethylation, and demethylated paraquat was found nontoxic to Arabidopsis, clearly demonstrating paraquat degradation by a plant P450 enzyme. Our study revealed a novel molecular mechanism of paraquat resistance that has not been reported in plants. Our findings should provide a new means for developing paraquat resistance crops using AtPQT11/CYP86A4.

\section{Result}

\section{Activated expression of At1G01600 confers paraquat resistance in pqt11D}

From an Arabidopsis activation-tagging library of approximately 55000 lines, we isolated a number of paraquat tolerance mutants. One of them, pqt11D, displayed enhanced resistance to paraquat (Fig. S1A). We found that the T-DNA with four 35S enhancers next to the right border was inserted at $1646 \mathrm{bp}$ upstream the ATG codon of At1g01600 (Fig. S1B). As a result, the gene At1g01600 was activated (Fig, S1C), indicating that the activated expression of At $1 g 01600$ may be responsible for observed paraquat resistance in pqt11D. We therefore named At1g01600 as AtPQT11. 
AtPQT11 is predicted to encode CYP86A4, a cytochrome P450 enzyme with 554 amino acids. It is expressed in all tissues examined with relatively higher levels in roots, flowers and siliques (Fig. S1D), and induced by paraquat treatment for the first 3 hours, then declined (Fig. 1E).

The pqt11D phenotype can be recapitulated by overexpressing AtPQT11 in the

To confirm whether the paraquat resistance of pqt11D was caused by an activated expression of AtPQT11, we generated two AtPQT11 overexpression lines (OX1-4 and OX3-1) and obtained a knockout mutant pqt11 (Salk_073078), in which the T-DNA was inserted in the first exon of AtPQT11 from ABRC (Fig. S1B), and confirmed all the genetic materials by 3-primers PCR (Fig. S1F) and qRT-PCR analyses (Fig. S1G).

Seed germination assay in response to paraquat treatment showed that two OX lines had significantly higher survival after 7 days on MS medium with $2 \mu \mathrm{M}$ paraquat compared with the wild type, while the knockout mutant pqt11 became hypersensitive (Fig. 1A and B).

We also conducted paraquat resistance assay on soil-grown plants of different AtPQT11 genotypes. When the plants grew to the stage with 8 rosette leaves, they were sprayed with $10 \mu \mathrm{M}$ paraquat every 3 days. 10 days after the first spray, The $\mathrm{OX}$ lines showed a significantly higher survival than wild type control, whereas the knockout mutant exhibited sicker phenotype and lower survival (Fig. 1C and D).

Together, these results demonstrate that an elevated expression of AtPQT11 confers paraquat resistance in Arabidopsis. cDNA into pET28a vector (pET28a-P450) and expressed in E. coli. Based on 3-D simulation of AtPQT11 structure (Fig. S2), we created single mutations of three residues G309A, S461A, G463A around the predicted substrate binding pocket and further expressed these three mutants in E. coli, respectively. In the absence of paraquat in liquid culture medium, there was no difference was observed between the 
expressing pET28a-P450 displayed a remarkable resistance to $1 \mathrm{mM}$ paraquat than those harboring the empty pET28a, whereas the bacteria transformed with the constructs carrying 3 different point mutations in AtPQT11 exhibited no paraquat resistance as the empty vector control (Fig. 1E). When grew on solid agar medium containing $1 \mathrm{mM}$ paraquat, only bacteria expressing pET28a-P450 formed colony plaques (Fig. 1F). These results further confirm the role of AtPQT11 in paraquat resistance and indicate that the amino acid residues are critical for its function.

\section{AtPQT11 catalyzes the conversion of paraquat to $\mathrm{N}$-demethyl paraquat in vitro}

Considering that AtPQT11 encodes a P450 protein, we reasoned AtPQT11 might catabolize paraquat to a nontoxic product. To demonstrate this, we first established an in vitro enzyme assay using E. coli expressed AtPQT11 protein, paraquat as substrate, and NADPH as electron donor as described in Materials and Methods. The consumption of NADPH provides a convenient spectrophotometric monitor at $\mathrm{OD}_{340 \mathrm{~nm}}$ of the reaction. As shown in Fig. 2A, the consumption of NADPH was AtPQT11-dependent in the reaction and increased with incubation time. Therefore, we established the in vitro enzyme assay for AtPQT11 and used this assay to determine the enzyme kinetic properties (Fig. 2B-D).

To identify the product of the reaction, the paraquat metabolite of the in vitro reaction was analyzed by LC-MS/MS. A product peak was found at $2.7 \mathrm{~min}$ with $\mathrm{m} / \mathrm{z}$ 171, which was AtPQT11- and NADPH-dependent (Fig. 3A-D, left panels). To confirm that this product is derived from paraquat, we used the ${ }^{2} \mathrm{H}$-labeled paraquat as the substrate, whose eight hydrogen atoms on paraquat pyridine ring were replaced by deuterium so that the $\mathrm{m} / \mathrm{z}$ of paraquat changed from 186 to 194 . As expected, we found the product peak with m/z 179, which was also AtPQT11- and NADPH-dependent (Fig. 3A-D, right panels ). The reaction product has the same mass as N-demethyl paraquat (m/z 171).

To resolve the identity of the product, we analyzed the MS/MS spectrum and found that the product showed the same fragment ions as N-demethyl paraquat standard (Fig. 3F, left panel and G, right panel), which was further confirmed by the same experiments with ${ }^{2} \mathrm{H}$-labeled paraquat (Fig. 3E and F, right panels). These 
results unequivocally show that the product is derived from the substrate and the product matches $\mathrm{N}$-demethyl paraquat based on their fragment ion pattern (Fig. 3G). Thus, we have demonstrated that AtPQT11/ CYP86A4 functions as an N-demethylase capable of converting paraquat to $\mathrm{N}$-demethyl paraquat.

\section{$\mathrm{N}$-demethyl paraquat is nontoxic to Arabidopsis}

Have identified N-demethyl paraquat as the product, we decided to find out whether N-demethyl paraquat has herbicide activity. To demonstrate this, we simply tested whether the N-demethyl paraquat is toxic to Arabidopsis. Seed germination assay in the presence of $\mathrm{N}$-demethyl paraquat showed that two OX lines, WT and knockout mutant pqt11 all survived and grew well on MS medium with $2 \mu \mathrm{M}$ $\mathrm{N}$-demethyl paraquat or $2 \mu \mathrm{M}$ bipyridine (double demethylated paraquat) compared with paraquat (Fig. 4A and B). This result clear shows that $\mathrm{N}$-demethyl paraquat and bipyridine are nontoxic to Arabidopsis. Therefore, we have uncovered the molecular mechanism underlying the paraquat resistance of pqt11D.

\section{Discussion}

In this study we reported the paraquat resistance mutant pqt11D and the underlying molecular mechanism. The phenotype of the mutant was caused by an activated expression of AtPQT11, which was confirmed by recapitulation analyses in Arabidopsis wild type as well as in E. coli (Fig. 1 and Fig. S1A-C). The paraquat hypersensitive phenotype of the AtPQT11 knockout mutant also supports that AtPQT11 is responsible for the observed paraquat resistance (Fig. 1 and 4). Further analyses revealed the AtPQT11 catalyzed biochemical reaction converting paraquat to single demethylated product (Fig. 2 and 3), and we also found that N-demethyl paraquat is nontoxic to Arabidopsis (Fig. 4).

CYP450 enzymes is widely found in biosynthesis or catabolic processes through the whole life in all living organisms ${ }^{22}$. Several members of CYP450 family have been reported to detoxify herbicides ${ }^{23,24}$. As a xenobiotic, paraquat is not a natural substrate of the CYP450 enzyme AtPQT11. The natural substrates of 
AtPQT11 remain unknown at present. AtPQT11 can catalyze the conversion of paraquat to N-demethyl paraquat perhaps because paraquat may have similar structural resemblance to its natural substrates.

Since paraquat is a widely used herbicide in agriculture, paraquat resistant weeds have emerged, which is a potential threat to agriculture. Generally, plants could gain paraquat resistance in three ways. First, paraquat absorption and transport are limited, thus preventing paraquat from reaching chloroplasts. Second, an enhanced intracellular antioxidant capacity would help plants to scavenge ROS more efficiently. Third, paraquat may be degraded to nontoxic metabolites. Plants may have these strategies in single or in combination to produce paraquat resistance ${ }^{25}$. There are many reports for the first two strategies $4,10,13,15,16$. However, paraquat degradation by plants has not been reported thus far. Our finding with pqt11D fills up the gap for the third strategy.

In conclusion, our findings with the paraquat resistant mutant pqt11D unequivocally demonstrate that AtPQT11, a member of the P450 super family detoxifies paraquat and confers paraquat resistance in Arabidopsis when overexpressed, therefore providing a much needed means to engineering paraquat resistance crops.

\section{Methods and materials}

\section{Plant material and growth conditions}

Arabidopsis Col-0 was used as wild type in this study. We also used Col-0 as the genetic background for all the mutants and transgenic plants. Salk_073078 was ordered from the Arabidopsis Biological Resource Center (ABRC). Seeds were sterilized in $10 \%$ bleach for 10 minutes and washed with sterile water 5 times. The washed seeds were kept in the dark at $4^{\circ} \mathrm{C}$ for 2 days before germination on MS medium at $22^{\circ} \mathrm{C}$ under $14 \mathrm{~h} \mathrm{light} / 10 \mathrm{~h}$ dark. 
Construction of AtPQT11 overexpression lines

In order to get overexpression lines, we used forward primer

5'-GGGGACAAGTTTGTACAAAAAAGCAGGCT

ATGGAAATATCCAATGCCATGC-3 and reverse primer

TTAAACCACTGCAACTCCCGTA-3' to obtain the CDS of CYP86A4. By means of

GATEWAY system $^{26}$, the CDS was cloned into vector pCB2004 via the shuttle vector of pDONR207.

The constructed plasmid was transformed into Agrobacterium tumefaciens

C58C1 by electroporation. The floral-dip method was used to transfer the construct

into plants ${ }^{27}$. The positive lines were screened as glufosinate resistant plants and homozygous lines were obtained from F2 population.

RNA extraction and qRT-PCR

We extracted the RNA from one-week-old seedlings by Trizol (Invitrogen, Carlsbad, California, USA) and reverse transcribed RNA to cDNA by TransScript Kit (TaKaRa). The expression of CYP86A4 was detected by RT-PCR or quantitative RT-PCR using forward primer 5'-CCCCAAGGGTTTCACTGAATTC-3' and reverse primer 5'-AAGTAAATGCGAAGCCTGCTTG -3' and Applied Biosystem Step One real-time PCR system or TaKaRa SYBR Premix Ex Taq II reagent kit. UBQ5 was used as the internal reference.

\section{Identification of the AtPQT11 knockout mutant} reverse primer 5'- AAATGTTGTCGAATGTGAGCC-3' and intermediate primer LBb1.3 5'-ATTTTGCCGATTTCGGAAC-3' were used to identify homozygotes of SALK_073078. The homozygotes showed only one band around $750 \mathrm{bp}$ while the wild type showed one band around $1000 \mathrm{bp}$. RT-PCR was performed to confirm null expression of the At1g01600 in SALK_073078 by using forward primer 5'ATGGAAATATCCAATGCCATGC-3' and reverse primer 5'- 
254

255

256

257

258

259

260

261

262

263

264

265

266

267

268

269

270

271

272

273

\section{Seed germination assay}

Sterilized seeds for each line including WT, knock out mutant SALK_073078 (pqt11/cyp86a4) and two overexpression lines OX1-4 and OX3-1 were treated at $4^{\circ} \mathrm{C}$ in dark condition for 2 days. Then we put these seeds on MS medium with $2 \mu \mathrm{M}$ paraquat, MS medium was used as control. The plates were placed at $22^{\circ} \mathrm{C}$ under 14 $\mathrm{h}$ light/10 h dark cycles. We considered the appearance of two green cotyledons as successful germination, 1 week later we recorded germination $\%$.

\section{Paraquat resistance assay on soil-grown plants}

One-week-old seedlings grown on MS medium were transferred to soil. We planted 5 seedlings per pot, grew to the stage with 8 rosette leaves, and sprayed with $10 \mu \mathrm{M}$ paraquat and sprayed again 3 days later. Photos and survival rate were recorded 10 days after the first spray.

\section{3-D model of At PQT11 structure}

The 3-D structure model of CYP86A4 was produced by Phyre2 and paraquat was docked into the structure model. Residues within the distance of $4 \AA$ around paraquat are colored in red. Conservative residues likely responsible for the binding of paraquat are labeled and chosen as mutation sites.

\section{Site-directed mutagenesis of AtPQT11}

Specific primers for site-directed mutagenesis were designed, for changing 309Gly to Ala, 461Cys to Ala, 463Gly to Ala as listed in Table S1. pET28-CYP86A4 or pCB2004-CYP86A4 were set as template and the PCR procedure was set for 20 cycles. After the PCR products were recycled, $1 \mu \mathrm{l}$ Dpnlwas added to digest these products. Then transferred these products to DH5 $\alpha$ and extracted plasmids from overnight culture.

\section{Paraquat resistance assay in $E$. coli}

We used Rosetta or BL21 as host, transferred the constructed pET28-P450 and the three plasmids with point mutation: pET28-P450A (Gly309Ala), pET28-P450B (Cys461 Ala) and pET28-P450C (Gly463Ala) into the host bacteria, and empty vector pET28a as control. Single colonies grown on the plate were picked and cultured 
overnight. Then the overnight culture was diluted into fresh LB medium with a starting $\mathrm{OD}_{600}$ value of 0.1 , and paraquat was added to make its final concentration of $1 \mathrm{mM}$. Another set of culture with $0 \mathrm{mM}$ paraquat was used as control. The $\mathrm{OD}_{600}$ value of the culture was monitored at regular intervals for 36 hours. Each culture was triplicated and the growth curves were obtained. When tested on solid LB plate with 1 $\mathrm{mM}$ paraquat, the overnight liquid bacteria culture was inoculated on the plate with 0 or $1 \mathrm{mM}$ paraquat and incubated overnight at $37^{\circ} \mathrm{C}$.

\section{Bacterial expression of AtPQT11}

The CDS of AtPQT11 was ligated to the vector pET28a by T4 ligase. The constructed plasmid was transformed into Rosetta strain with kanamycin selection. A clone was picked from medium and was added with liquid $\mathrm{LB}$, when the $\mathrm{OD}_{600}$ of medium reached 0.6 , IPTG was added with a final concentration of $0.3 \mathrm{mM}$. The culture was shaken in $16^{\circ} \mathrm{C}$ with the speed of $120 \mathrm{rpm}$ for $12-16 \mathrm{~h}$. The culture was centrifuged for $10 \mathrm{~min}$ at $4500 \mathrm{rpm}$ to harvest bacteria, then the bacterial pellet was resuspended with protein isolation buffer $(20 \mathrm{mM}$ Tris-Cl pH7.5, $0.2 \mathrm{M} \mathrm{NaCl}, 5 \%$ glycerin, 1 mM EDTA, 1 mM PMSF) + 1\% Dodecyl-beta-D-maltoside (m/v), and ultrasonicated to break bacterial cell walls. The cell lysate was rotated at $4^{\circ} \mathrm{C}$ for $2 \mathrm{~h}$ and then centrifuged at $45000 \mathrm{rpm}$ for $30 \mathrm{~min}$. The supernatant was collected for AtPQT11 protein purification using Ni-IDA-sefinose resin kit.

\section{In vitro enzyme assay of AtPQT11}

The reaction mixtures contained $0.1-10 \mu \mathrm{M}$ paraquat as substrate, $50-100 \mu \mathrm{g}$ AtPQT11/ CYP86A4 protein, $1 \mathrm{mM}$ NADPH as electron donor, $25 \mathrm{mM}$ phosphate buffer (pH7.0) in a total volume of $50 \mu$. The reaction was initiated by adding NADPH. The reaction mixtures without NADPH or AtPQT11/CYP86A4 were set as controls. The mixtures were incubated for $30 \mathrm{~min}$ at $27^{\circ} \mathrm{C}$ and terminated by adding $50 \mu \mathrm{l}$ acetonitrile. To determine the enzyme kinetic properties, the reaction was spectrophotometrically monitored at $340 \mathrm{~nm}$ for NADPH consumption. To identify the reaction product, the mixtures was centrifuged at $14000 \mathrm{~g}$ for $20 \mathrm{~min}$, and the 
supernatant was analyzed by HPLC-MS/MS as described below.

312

\section{HPLC-MS/MS}

HPLC-MS/MS analysis was performed using Agilent Q-TOF-LC/MS 6545 device (Agilent Technologies, Palo Alto, CA, USA) . A XBridge BEH HILIC 2.5 $\mu \mathrm{m}$ Column $(2.1 * 100 \mathrm{~mm})$ from Waters was used for chromatographic separation. The mobile phase was a mixture of solvent $\mathrm{A}(10 \mathrm{mM}$ ammonium acetate added with $0.1 \%$ $\mathrm{V} / \mathrm{V}$ formic acid) and solvent $\mathrm{B}(10 \mathrm{mM}$ ammonium acetate dissolved in $95 \%$ acetonitrile) and was delivered at a flow rate of $0.3 \mathrm{~mL} / \mathrm{min}$. The column temperature was set at $40^{\circ} \mathrm{C}$ and the injection volume was $2 \mu$. The sample was followed the gradient: $90 \%$ B (0 min), 10\% B (3 min), 1\% B (5 min), 90\% B (5 min), with a total run time of $12 \mathrm{~min}$.

Mass acquisition was performed in the positive ionization mode at a fragmentation voltage of $175 \mathrm{~V}$. The following parameters were used: drying gas flow, $8 \mathrm{~L} / \mathrm{min}$; temperature, $325^{\circ} \mathrm{C}$; sheath gas flow, $11 \mathrm{~L} / \mathrm{min}$; temperature, $350{ }^{\circ} \mathrm{C}$; nebulizer pressure, $45 \mathrm{psi}$; and capillary voltage, $3500 \mathrm{~V}$. The collision energy was set at $15-40 \mathrm{~V}$, and the mass range was recorded from $\mathrm{m} / \mathrm{z}$ 50-1700. The acquired MS and MS/MS data files were analyzed using Agilent MassHunter Qualitative Analysis B.07.00.

\section{Toxicity assay of $\mathrm{N}$-demethyl paraquat}

Seeds of different genotypes were washed and treated at $4^{\circ} \mathrm{C}$ in dark condition for 2 days, then plated on MS medium with $2 \mu \mathrm{M} N$-demethyl paraquat, $2 \mu \mathrm{M}$ bipyridine, $2 \mu \mathrm{M}$ paraquat, respectively. MS medium was used as mock. Germination and survival was recorded after 1 week.

\section{Statistical analyses}

Statistical significance was evaluated at the 0.05 probability level using Student's t-test.

\section{Supplemental information}

Figure S1. Paraquat resistance phenotype of pqt11D and confirmation of 
AtPQT11 expression in pqt11, pqt11D, and overexpression lines.

Fig. S2. 3-D model of AtPQT11 structure.

Table S1. Primers used in this study.

\section{Acknowledgements}

This work was supported by grants from the National Natural Science Foundation

344 of China (grant no. 31770273). We thank ABRC for providing Arabidopsis mutant

345 seeds.

346 Author's Contributions

$347 \quad \mathrm{CBX}$ and YJH designed the experiments. YJH. performed the major experiments.

348 YPH isolated pqt11D and identified the T-DNA insertion site. JQX, WTH, AMM,

349 XQQ, YXC, participated in experiments and data analyses. ZPF, YFC, YiPH, LPG

350 contributed to LC-MS/MS analyses. YJH wrote the manuscript. CBX and LPG

351 revised the manuscript and supervised the project.

352 Conflict Interests

353 The authors declare no conflict of interest. 


\section{References}

Baldwin, B., Clarke, C. \& Wilson, I. Paraquat in chloroplasts. Biochimica et Biophysica Acta (BBA)-Bioenergetics 162, 614-617 (1968). Farrington, J., Ebert, M., Land, E. \& Fletcher, K. Pulse radiolysis studies of the reaction of paraquat radical with oxygen. Implication for the mode of action of bipuridyl herbicides. Biochimica et Biophysica Acta 314, 372-381 (1973). Lewthwaite, S. \& Triggs, C. Identification of paraquatresistant Solanum nigrum and $\mathrm{S}$ americanum biotypes. New Zealand Plant Protection 62, 349-355 (2009). Cronq. Pesticide Biochemistry and Physiology 88, 57-65, doi:10.1016/j.pestbp.2006.08.013 (2007).

Lasat, M. M., DiTomaso, J. M., Hart, J. J. \& Kochian, L. V. Evidence for vacuolar sequestration of paraquat in roots of a paraquat-resistant Hordeum glaucum biotype. Physiologia Plantarum 99, 255-262 (1997).

Hawkes, T. R. Mechanisms of resistance to paraquat in plants. Pest Manag Sci 70, 1316-1323, doi:10.1002/ps.3699 (2014).

Shaaltiel, Y. \& Gressel, J. Kinetic analysis of resistance to paraquat in Conyza: evidence that paraquat transiently inhibits leaf chloroplast reactions in resistant plants. Plant physiology $\mathbf{8 5}$, 869-871 (1987).

Ye, B. \& Gressel, J. Transient, oxidant-induced antioxidant transcript and enzyme levels correlate with greater oxidant-resistance in paraquat-resistant Conyza bonariensis. Planta 211, 50-61 (2000).

Tsuji, K., Hosokawa, M., Morita, S., Miura, R. \& Tominaga, T. Resistance to paraquat in M azus pumilus. Weed Research 53, 176-182 (2013).

Chen, R. et al. The Arabidopsis PARAQUAT RESISTANT2 gene encodes an S-nitrosoglutathione reductase that is a key regulator of cell death. Cell Research 19, 1377-1387, doi:10.1038/cr.2009.117 (2009).

Kurepa, J., Smalle, J., Va, M., Montagu, N. \& Inzé, D. Oxidative stress tolerance and longevity in Arabidopsis: the late-flowering mutant gigantea is tolerant to paraquat. The Plant Journal 14, 759-764 (1998).

Fujita, M. et al. Natural variation in a polyamine transporter determines paraquat tolerance in Arabidopsis. Proceedings of the national academy of sciences of the united states of america 109, 6343-6347, doi:10.1073/pnas.1121406109 (2012).

intracellular transport of paraquat. Plant Physiology 162, 470-483, doi:10.1104/pp.113.213892 (2013).

Soar, C., Preston, C., Karotam, J. \& Powles, S. Polyamines can inhibit paraquat toxicity and translocation in the broadleaf weed Arctotheca calendula. Pesticide Biochemistry and Physiology 80, 94-105 (2004). 
AtPQT11 by YJ Huang et al.

396

397

398

399

400

401

402

403

404

405

406

407

408

409

410

411

412

413

414

415

416

417

418

419

420

421

422

423

424

425

426

$427 \quad 26$

428

429

430

431

432

433

434

435

436

$15 \mathrm{Xi}$, J., Xu, P. \& Xiang, C. B. Loss of AtPDR11, a plasma membrane-localized ABC transporter, confers paraquat tolerance in Arabidopsis thaliana. Plant Journal 69, 782-791, doi:10.1111/j.1365-313X.2011.04830.x (2012).

16 Luo, C. et al. PARAQUAT TOLERANCE3 Is an E3 Ligase That Switches off Activated Oxidative Response by Targeting Histone-Modifying PROTEIN METHYLTRANSFERASE4b. PLOS Genetics 12 (2016).

17 Sipari, N., Lihavainen, J., Shapiguzov, A., Kangasjärvi, J. \& Keinänen, M. Primary Metabolite Responses to Oxidative Stress in Early-Senescing and Paraquat Resistant Arabidopsis thaliana rcd1 (Radical-Induced Cell Death1). Frontiers in plant science 11, 194 (2020).

18 Schuler, M. A. \& Werck-Reichhart, D. Functional genomics of P450s. Annual review of plant biology 54, 629-667 (2003).

19 Kreuz, K., Tommasini, R. \& Martinoia, E. Old enzymes for a new job (herbicide detoxification in plants). Plant physiology 111, 349 (1996).

20 Didierjean, L. et al. Engineering herbicide metabolism in tobacco and Arabidopsis with CYP76B1, a cytochrome P450 enzyme from Jerusalem artichoke. Plant Physiology 130, 179-189 (2002).

21 Yamada, T., Kambara, Y., Imaishi, H. \& Ohkawa, H. Molecular Cloning of Novel Cytochrome P450 Species Induced by Chemical Treatments in Cultured Tobacco Cells. Pesticide Biochemistry and Physiology 68, 11-25 (2000).

22 Mansuy, D. The great diversity of reactions catalyzed by cytochromes P450. Comp Biochem Physiol C Pharmacol Toxicol Endocrinol 121, 5-14, doi:10.1016/s0742-8413(98)10026-9 (1998).

23 Xiang, W., Wang, X. \& Ren, T. Expression of a wheat cytochrome P450 monooxygenase cDNA in yeast catalyzes the metabolism of sulfonylurea herbicides. Pesticide biochemistry and physiology 85, 1-6 (2006).

24 Siminszky, B., Corbin, F. T., Ward, E., Fleischmann, T. J. \& Dewey, R. E. Expression of a soybean cytochrome P450 monooxygenase CDNA in yeast and tobacco enhances the metabolism of phenylurea herbicides. Proceedings of the National Academy of Sciences of the United States of America 96, 1750-1755 (1999).

25 Baucom, R. S. The remarkable repeated evolution of herbicide resistance. Am J Bot 103, 181-183, doi:10.3732/ajb.1500510 (2016).

26 Lei, Z. et al. High-throughput Binary Vectors for Plant Gene Function Analysis. Journal of Integrative Plant Biology 49, 556-567 (2007).

27 Clough, S. J. \& Bent, A. F. Floral dip: A simplified method for Agrobacterium-mediated transformation of Arabidopsis thaliana. Plant Journal 16, 735-743 (1998). 
bioRxiv preprint doi: https://doi.org/10.1101/2021.06.23.449549; this version posted June 23, 2021. The copyright holder for this preprint (which was not certified by peer review) is the author/funder. All rights reserved. No reuse allowed without permission.

AtPQT11 by YJ Huang et al.

437

438

439 


\section{Figure Legends}

Figure 1. AtPQT11 confers paraquat resistance in Arabidopsis and E. coli when overexpressed.

(A) The seeds of wild type (WT), AtPQT11 overexpression lines (OX1-4, OX3-1), before survival was recorded. 100 seeds were used for each genotype. 3 replicates were performed for each treatment. Bar $=1 \mathrm{~cm}$.

(B) Survival \% in germination as in (A). Values are mean $\pm \mathrm{SD}(\mathrm{n}=3)$. The low case letters indicate significant differences $(\mathrm{P}<0.05)$. (C) Phenotypes of wild type (WT), AtPQT11 overexpression lines (OX1-4, OX3-1), and pqt11 grown in the soil. When plants grew to 8-rosette-leaf stage, they were sprayed with 0 (control) or $10 \mu \mathrm{M}$ paraquat and sprayed again 3 days later. Photos and survival \% were recorded 10 days after the first spray. 40 plants were used for each genotype. 3 replicates were performed for each treatment.

(D) Survival $\%$ as in $(C)$. Values are mean $\pm \mathrm{SD}(\mathrm{n}=3)$. The low case letters indicate significant differences $(\mathrm{P}<0.05)$.

(E) Bacterial growth curves. Plasmids pET28a-AtPQT11 (pET28a-P450) with different changed residue: pET28-P450A (Gly309Ala), pET28-P450B (Cys461Ala) and pET28-P450C (Gly463Ala) were transferred into Rosetta, and cultured in LB medium with 0 or $1 \mathrm{mM}$ paraquat. The growth of the cultures with initial $\mathrm{OD}_{600}$ adjusted to 0.1 was monitored for 36 hours. 3 replicates were performed for each treatment. Values are mean $\pm \mathrm{SD}(\mathrm{n}=3)$.

(F) Bacterial growth on solid agar plates. Bacterial strains as in (E) were inoculated on solid LB medium with 0 or $1 \mathrm{mM}$ paraquat and incubated overnight at $37^{\circ} \mathrm{C}$.

\section{Figure 2. In vitro kinetic parameters of AtPQT11}

467 (A) AtPQT11-dependent reaction. The enzyme assay was carried out as described in 
AtPQT11. Values are mean \pm SD $(n=3)$.

(B) Michaelis-Menton plot for paraquat. $2 \mathrm{mM}$ NADPH was added to ensure it is in

472 excess in the reaction while varying the concentration of paraquat. Values are mean

$473 \pm \mathrm{SD}(\mathrm{n}=3)$.

474 (C) Michaelis-Menton plot for NADPH. $2 \mathrm{mM}$ Paraquat was added to ensure it is in excess in the reaction while varying the concentration of NADPH. Values are mean $\pm \mathrm{SD}(\mathrm{n}=3)$.

(D) The enzyme kinetics parameters. $V_{\max }$ and $K_{m}$ were obtained from (B) and (C), $K_{\mathrm{m}}$ and $V_{\max }$ values were estimated by using non-linear regression analysis.

479

480

481

\section{Figure 3. Identification of the reaction product by LC-MS/MS.}

(A-D) The reaction was carried out with paraquat or ${ }^{2} \mathrm{H}$-paraquat as substrate as described in Materials and Methods for 30 min. The reaction supernatant was subjected to LC-MS/MS analysis by scanning product at M/Z 171 or 179. (A) and (B) are the reaction for 0 or $30 \mathrm{~min},(\mathrm{C})$ and (D) are the reaction for $30 \mathrm{~min}$ but without NADPH or P450 enzyme.

(E-G) The MS/MS spectrum of substrate and product. (E) The MS/MS spectrum of paraquat when the reaction was just started. (F) The MS/MS spectrum of product when the reaction was completed (30 $\mathrm{min})$. (G) The MS/MS spectrum of paraquat standard and N-demethyl paraquat standard. MS/MS spectrum of the product closely matches with that of $\mathrm{N}$-demethyl paraquat (M/Z 171).

\section{Figure 4. N-demethyl paraquat is nontoxic to Arabidopsis.}

(A) Germination assay. The seeds of wild type (WT), AtPQT11 overexpression lines (OX1-4, OX3-1), and pqt11 were germinated on MS medium with 0 (Mock), $2 \mu \mathrm{M}$ paraquat (PQ), $2 \mu \mathrm{M} N$-demethyl paraquat (N-methyl PQ), or $2 \mu \mathrm{M}$ bipyridine, respectively for 1 week before survival was recorded. 100 seeds were used for each genotype. 3 replicates were performed for each treatment. Bar $=1 \mathrm{~cm}$.

(B) Survival \% in germination as in (A). Values are mean $\pm \mathrm{SD}(\mathrm{n}=3)$. The low case letters indicate significant differences $(\mathrm{P}<0.05)$. 
A
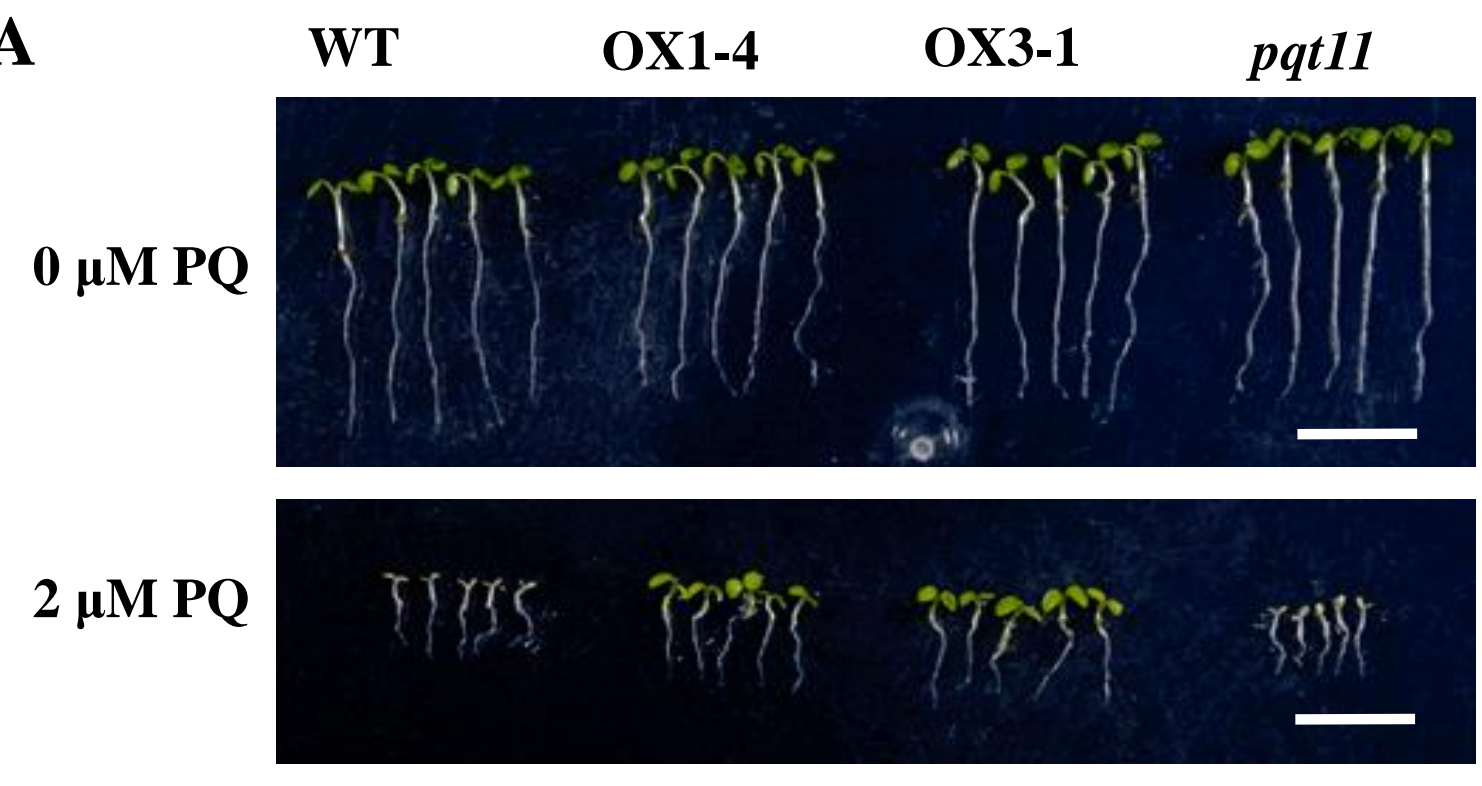

B

C
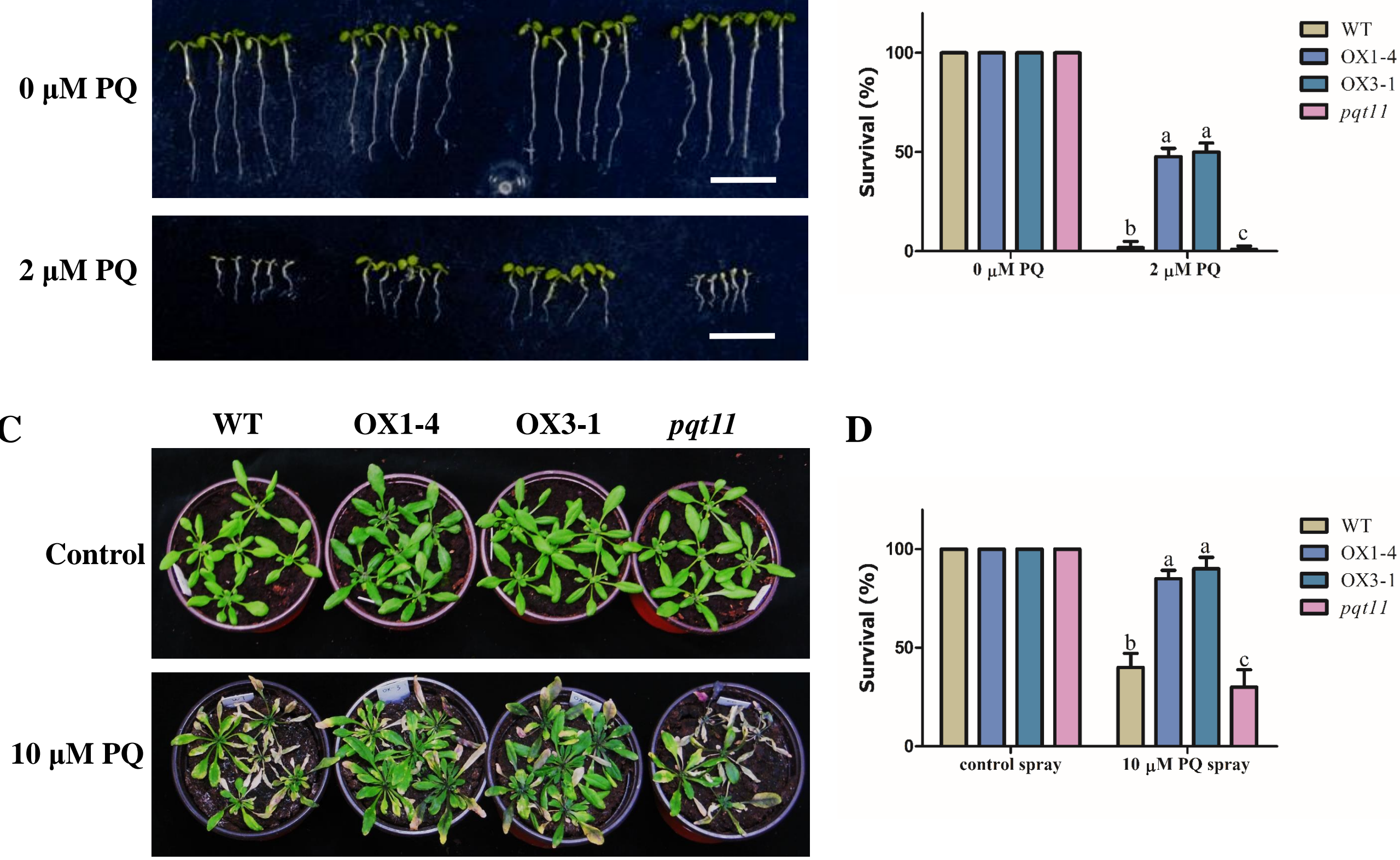

D

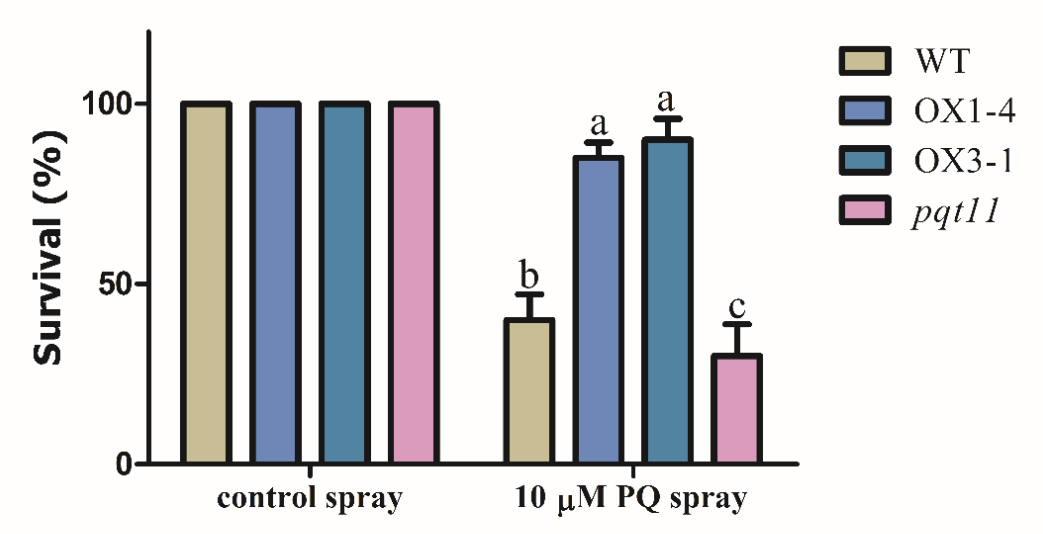

$\mathbf{E}$

$0 \mu \mathrm{M}$ PQ

$1 \mathrm{mM} P Q$
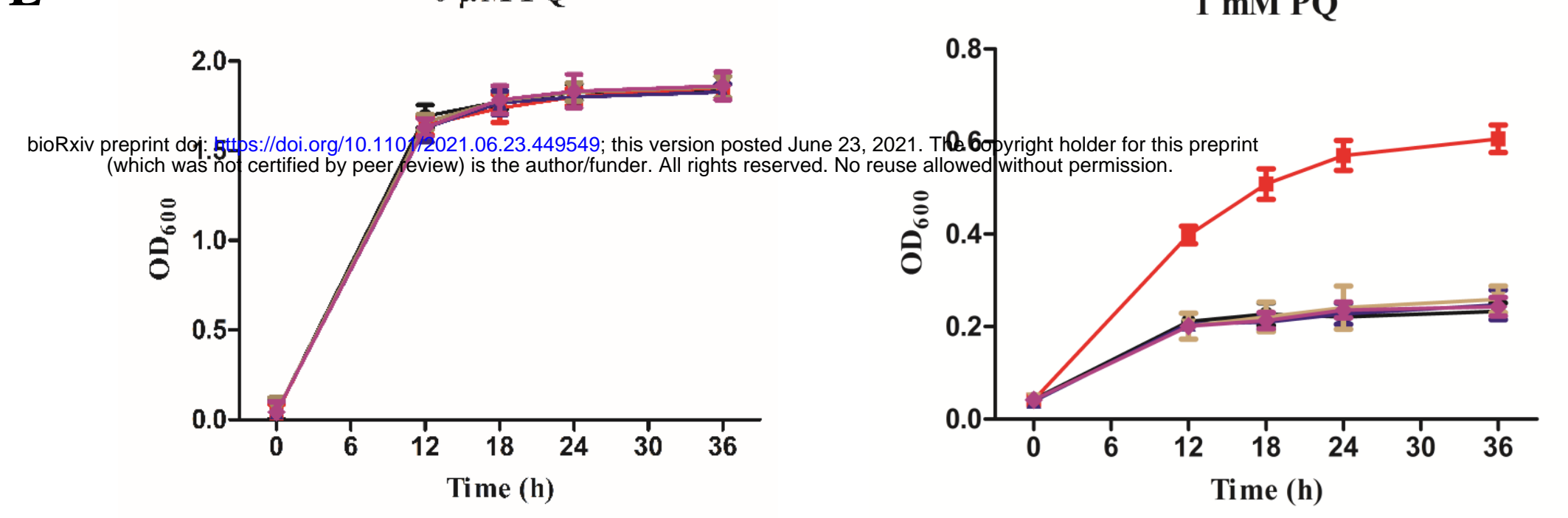

$\rightarrow$ pET28a

- pET28a-P450

$\leftarrow$ pET28a-P450A

$\rightarrow$ pET28a-P450B

$\neg$ pET28a-P450C

$\mathbf{F}$

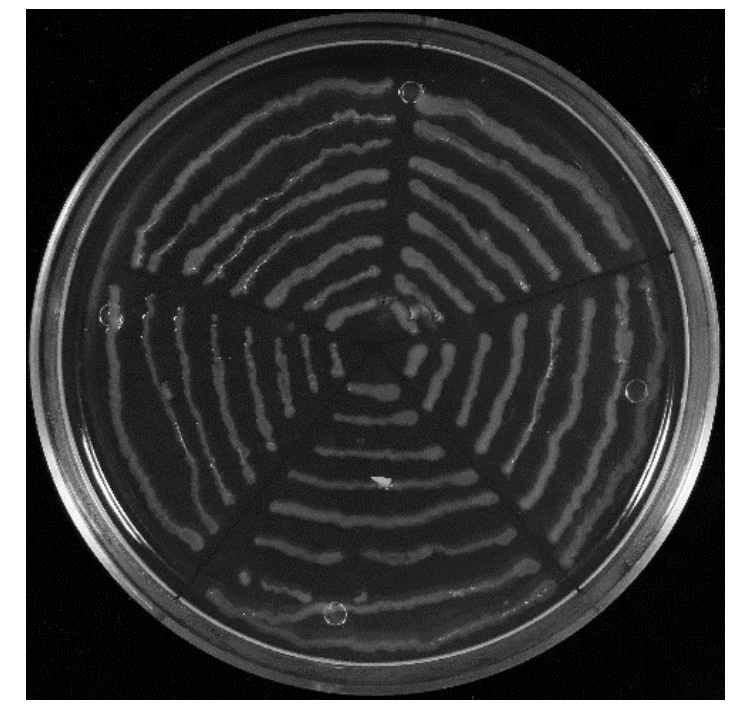

O mM PQ

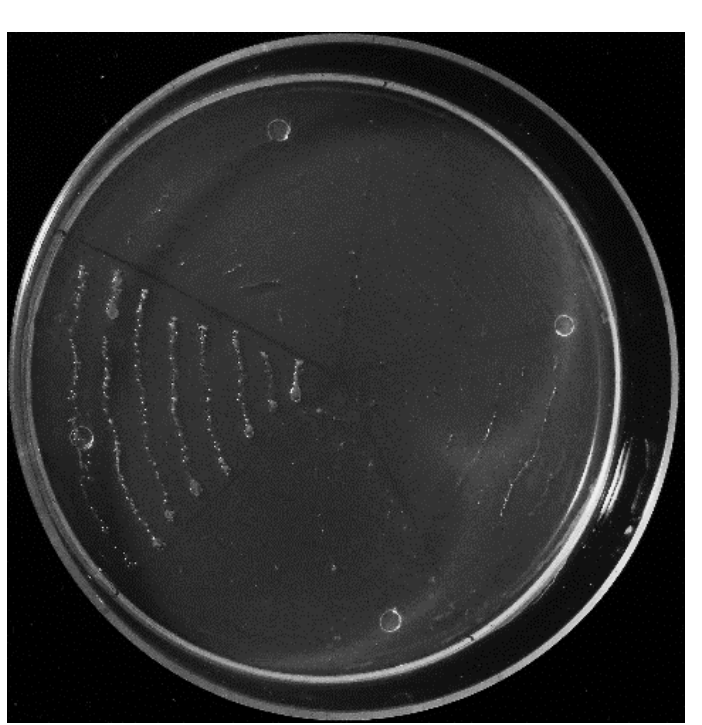

$1 \mathrm{mM}$ PQ

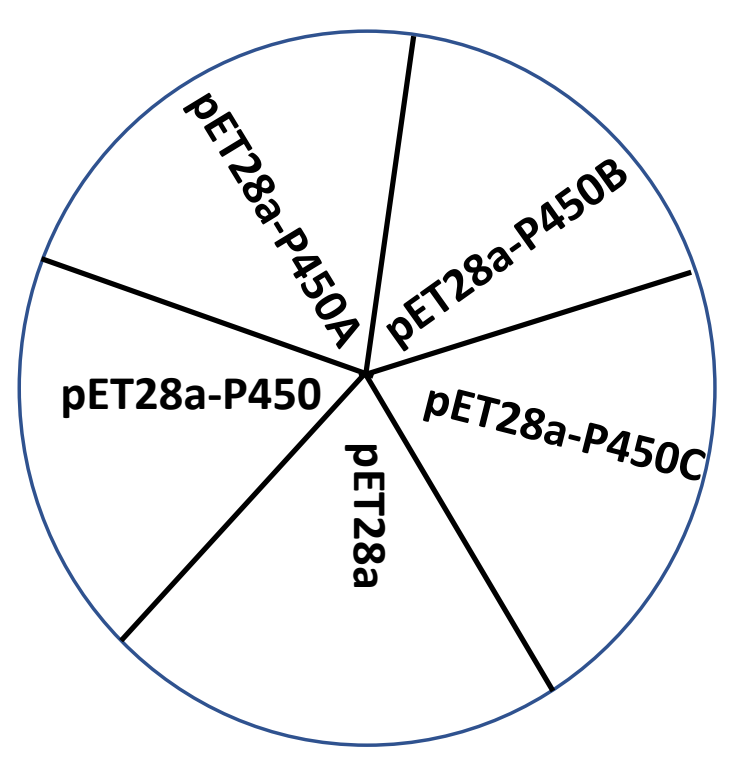

Figure 1. AtPQT11 confers paraquat resistance in Arabidopsis and E. coli when overexpressed.

(A) The seeds of wild type (WT), AtPQT11 overexpression lines (OX1-4, OX3-1), and pqt11 were germinated on MS medium with 0 or 2 $\mu \mathrm{M}$ paraquat (PQ) for 1 week before survival was recorded. 100 seeds were used for each genotype. 3 replicates were performed for each treatment. Bar $=1 \mathrm{~cm}$.

(B) Survival \% in germination as in (A). Values are mean $\pm \mathrm{SD}(\mathrm{n}=3)$. The low case letters indicate significant differences $(\mathrm{P}<0.05)$.

(C) Phenotypes of wild type (WT), AtPQT11 overexpression lines (OX1-4, OX3-1), and pqt11 grown in the soil. When plants grew to 8rosette-leaf stage, they were sprayed with 0 (control) or $10 \mu \mathrm{M}$ paraquat and sprayed again 3 days later. Photos and survival \% were recorded 10 days after the first spray. 40 plants were used for each genotype. 3 replicates were performed for each treatment.

(D) Survival \% as in (C). Values are mean $\pm \mathrm{SD}(\mathrm{n}=3)$. The low case letters indicate significant differences $(\mathrm{P}<0.05)$.

(E) Bacterial growth curves. Plasmids pET28a-AtPQT11 (pET28a-P450) with different changed residue (pET28a-P450A, pET28a-P450B, pET28a-P450C) were transferred into Rosetta, and cultured in LB medium with 0 or $1 \mathrm{mM}$ paraquat. The growth of the cultures with initial $\mathrm{OD}_{600}$ adjusted to 0.1 was monitored for 36 hours. 3 replicates were performed for each treatment. Values are mean $\pm \operatorname{SD}(\mathrm{n}=3$ ). (F) Bacterial growth on solid agar plates. Bacterial strains as in (E) were inoculated on solid LB medium with 0 or $1 \mathrm{mM}$ paraquat and incubated overnight at $37^{\circ} \mathrm{C}$. 
A

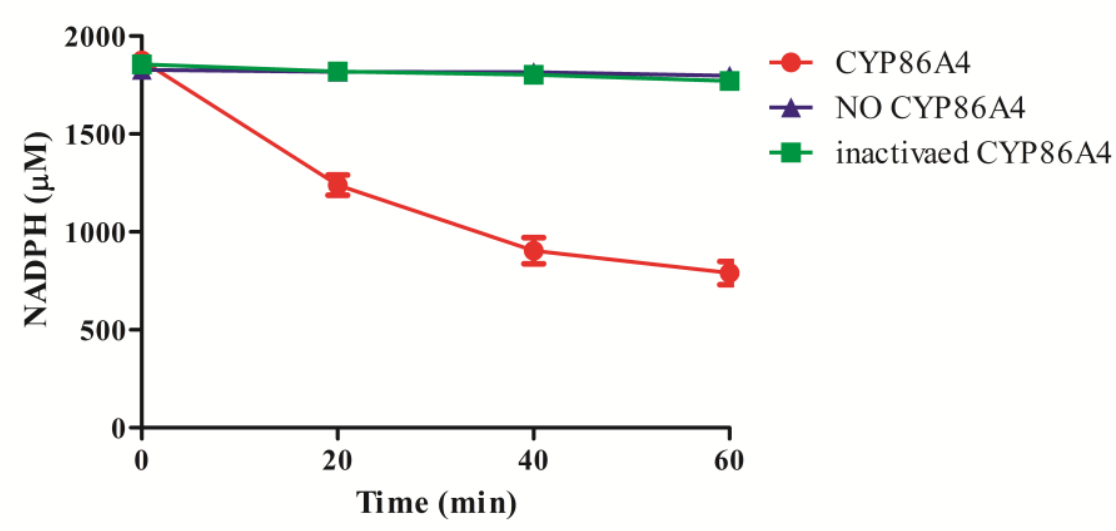

C

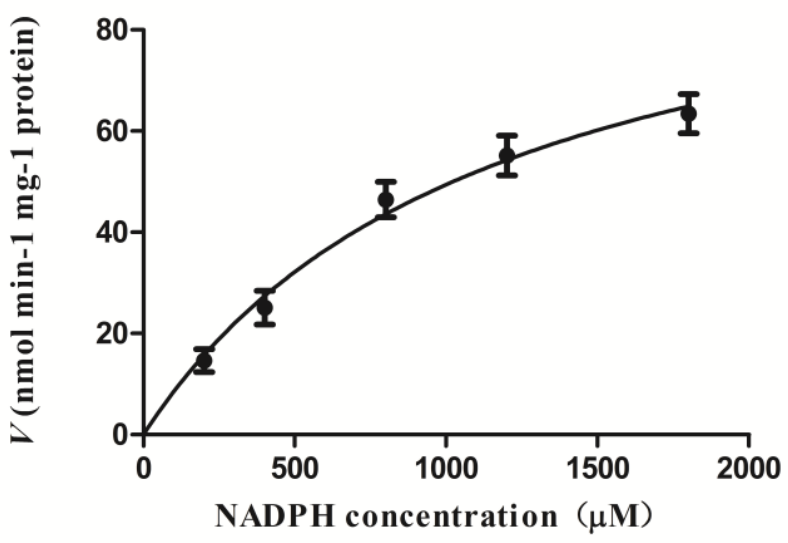

B

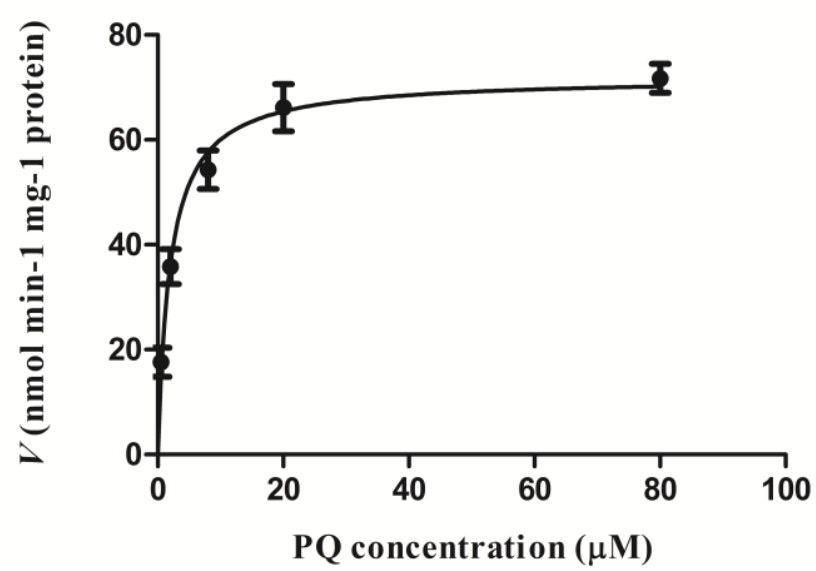

D

In vitro kinetic parameters of the CYP86A4 enzyme

\begin{tabular}{lcc}
\hline Substrate & $K_{\mathrm{m}}(\mu \mathrm{M})$ & $V_{\max }\left(\mathrm{nmol} \mathrm{min}{ }^{-1} \mathrm{mg}^{-1}\right.$ protein $)$ \\
\hline Paraquat & $1.34( \pm 0.21)$ & $71.91( \pm 2.29)$ \\
\hline NADPH & $1155( \pm 214.9)$ & $106.5( \pm 11.04)$ \\
\hline
\end{tabular}

\section{Figure 2. In vitro kinetic parameters of AtPQT11}

(A) AtPQT11-dependent reaction. The enzyme assay was carried out as described in Materials and Methods with 2 controls, no AtPQt11 (CYP684A) and inactivated AtPQT11. NADPH consumption was only observed in the reaction containing AtPQT11. Values are mean $\pm \mathrm{SD}(\mathrm{n}=3)$.

(B) Michaelis-Menton plot for paraquat. $2 \mathrm{mM}$ NADPH was added to ensure it is in excess in the reaction while varying the concentration of paraquat. Values are mean $\pm \mathrm{SD}(\mathrm{n}=3)$.

(C) Michaelis-Menton plot for NADPH. $2 \mathrm{mM}$ Paraquat was added to ensure it is in excess in the reaction while varying the concentration of NADPH. Values are mean \pm SD $(n=3)$.

(D) The enzyme kinetics parameters. $V_{\max }$ and $K_{m}$ were obtained from (B) and (C), $K_{\mathrm{m}}$ and $V_{\max }$ values were estimated by using non-linear regression analysis. 
Reaction with paraquat

A

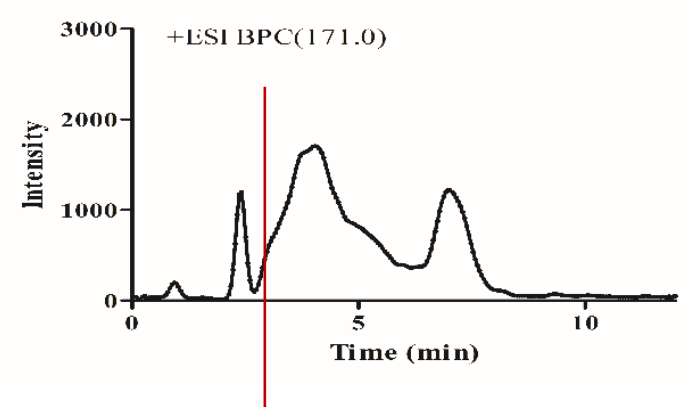

B

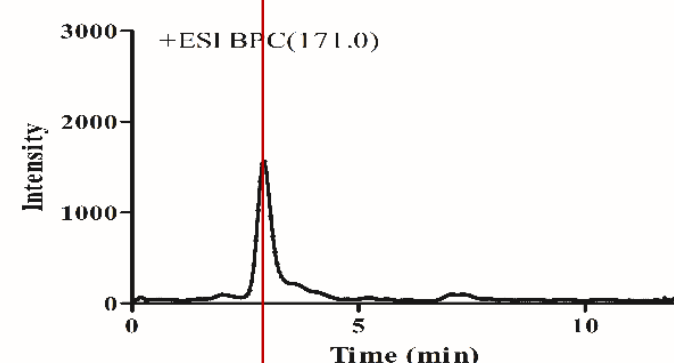

C NO NADPH 30 min

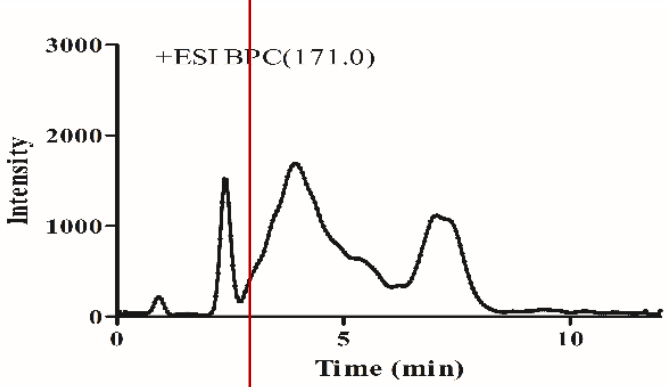

D

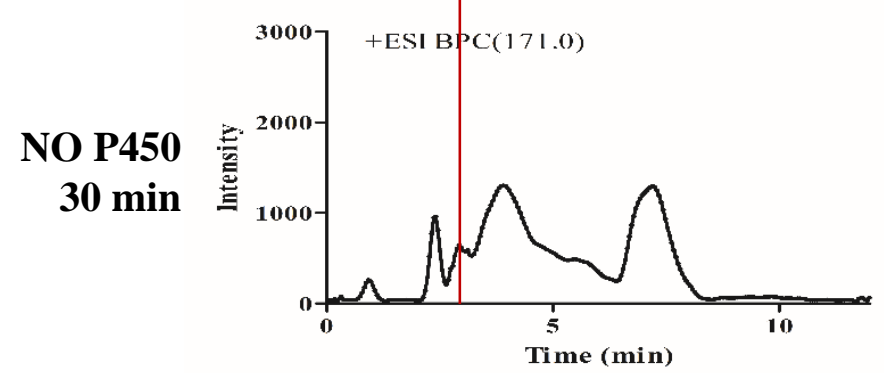

\section{Reaction with ${ }^{2} \mathrm{H}-P a r a q u a t$}
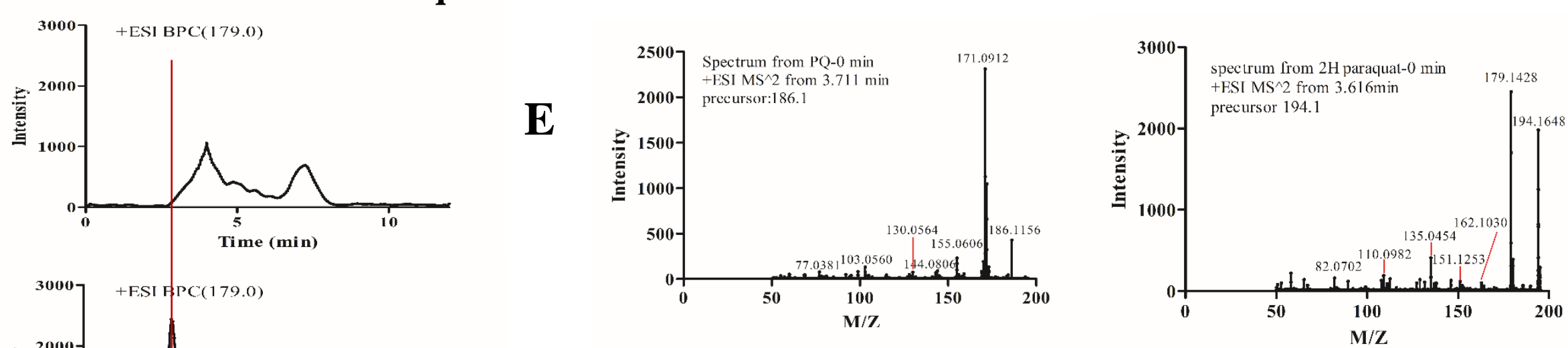

F
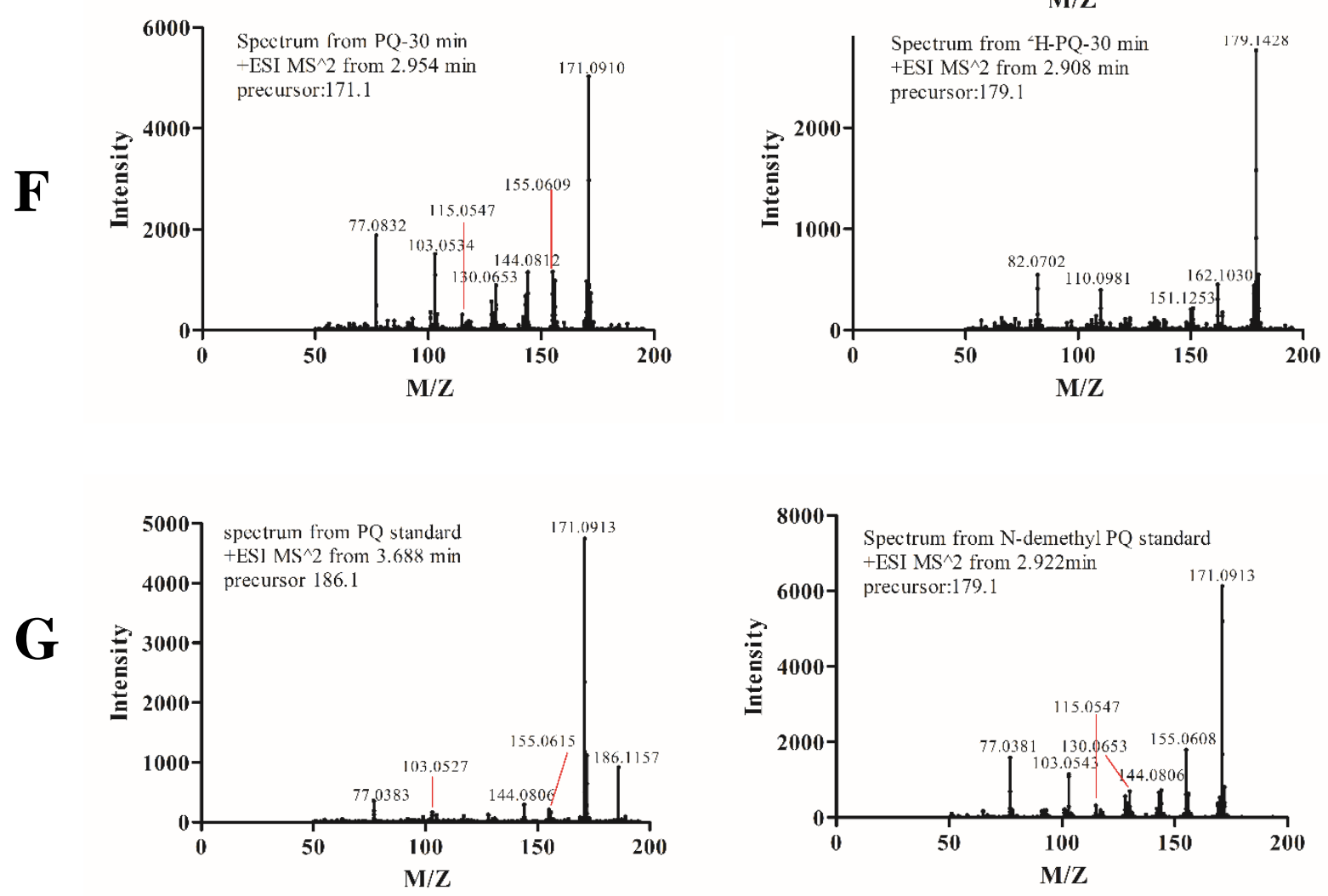

Figure 3. Identification of the reaction product by LC-MS/MS.

(A-D) The reaction was carried out with paraquat or ${ }^{2} \mathrm{H}$-paraquat as substrate as described in Materials and Methods for 30 min. The reaction supernatant was subjected to LC-

MS/MS analysis by scanning product at M/Z 171 or 179. (A) and (B) are the reaction for 0 or $30 \mathrm{~min}$, (C) and (D) are the reaction for 30 min but without NADPH or P450 enzyme.

(E-G) The MS/MS spectrum of substrate and product. (E) The MS/MS spectrum of paraquat when the reaction was just started. (F) The MS/MS spectrum of product when the reaction was completed (30 min). (G) The MS/MS spectrum of paraquat standard and N-demethyl paraquat standard. MS/MS spectrum of the product closely matches with that of $\mathrm{N}$-demethyl paraquat (M/Z 171). 


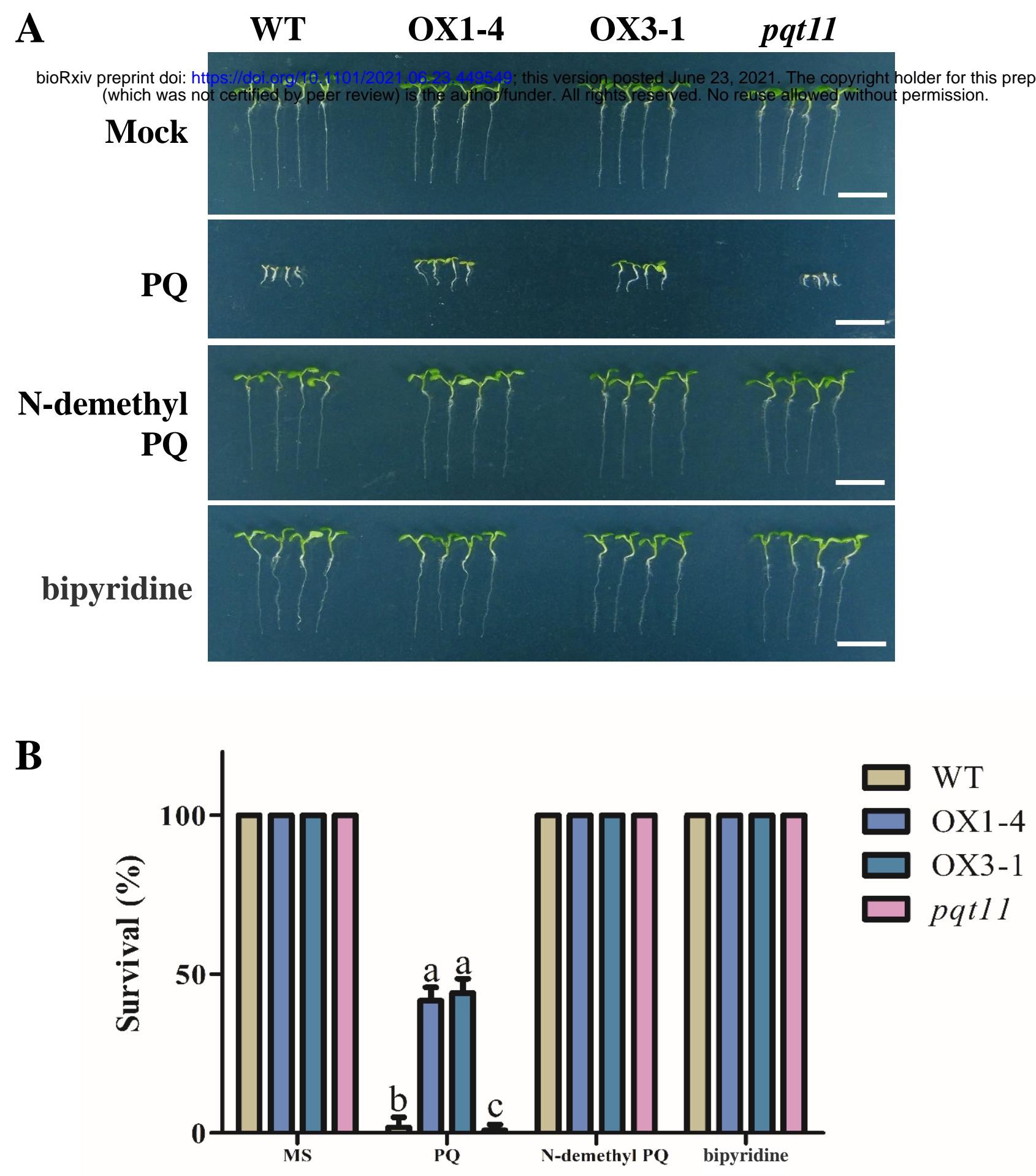

Figure 4. N-demethyl paraquat is nontoxic to Arabidopsis.

(A) Germination assay. The seeds of wild type (WT), AtPQT11 overexpression lines (OX1-4, OX3-1), and pqt11 were germinated on MS medium with 0 (Mock), $2 \mu \mathrm{M}$ paraquat (PQ), $2 \mu \mathrm{M}$ N-demethyl paraquat (N-methyl PQ), or $2 \mu \mathrm{M}$ bipyridine, respectively for 1 week before survival was recorded. 100 seeds were used for each genotype. 3 replicates were performed for each treatment. Bar $=1 \mathrm{~cm}$.

(B) Survival $\%$ in germination as in (A). Values are mean $\pm \operatorname{SD}(n=3)$. The low case letters indicate significant differences $(\mathrm{P}<0.05)$. 